\title{
Clonage du gène de susceptibilité au cancer du sein BRCA1, repérage du gène BRCA2: les firmes de biotechnologie en première ligne
}

En 1990, l'équipe de Mary-Claire King localisait un gène important de susceptibilité aux formes familiales de cancers du sein et de l'ovaire sur le bras long du chromosome 17 [1]. Ce gène fut appelé BRCA1. Dès lors, 7 à 8 groupes internationaux se mettaient en chasse de ce gène impliqué dans l'un des cancers les plus fréquents de l'espèce humaine. Dans les pays occidentaux, entre une femme sur neuf et une femme sur dix est appelée à avoir un cancer du sein dont l'issue reste trop souvent fatale. De 5 à $10 \%$ de ces cancers seraient familiaux et le gène $B R C A 1$ serait en cause dans environ le tiers ou la moitié de ces formes génétiques. La pénétrance du gène $B R C A 1$ est très grande puisque $90 \%$ des femmes ayant hérité d'un allèle anormal sont destinées à développer un cancer du sein au cours de leur vie. Compte tenu de l'importance de l'enjeu, notamment des perspectives commerciales offertes par les tests diagnostiques et les recherches thérapeutiques autorisées par la découverte du gène, plusieurs des équipes engagées dans la course au gène étaient des firmes privées de biotechnologie. C'est la société Myriad Genetics, en coopération avec l'université de l'Utah, qui vient de remporter le gros lot, sous la direction de Mark Skolnick, son principal dirigeant. Le gène s'étend sur plus de $100 \mathrm{~kb}$ et comporte 21 exons. Le transcrit principal mesure 7,8 kb et code pour une protéine de 1 1172 sède une région de type "doigt de zinc" dans sa partie amino-terminale et est particulièrement riche en résidus acides au niveau de son extrémité carboxyterminale, laissant supposer qu'elle pourrait être un facteur de transcription [2]. Dans huit familles affectées par des cancers héréditaires du sein, les auteurs ont trouvé trois mutations: deux mutations non-sens (un codon stop et un décalage de phase de lecture) et une mutation faux sens. Ces anomalies n'étaient observées dans aucune famille normale et ségrégaient, dans les familles à risques, avec la susceptibilité à la maladie. Dans une quatrième famille, l'anomalie observée était une absence d'expression d'un des allèles BRCA 1 . Ces différentes altérations suggérent que la susceptibilité aux cancers du sein et de l'ovaire est la conséquence d'une mutation avec perte de fonctions, comme cela est habituellement observé pour les autres anti-oncogènes : les malades possèdent une mutation germinale, héritée ou néomutation, d'un des allèles alors que l'autre allèle est inactivé dans la tumeur par un mécanisme quelconque, souvent une délétion.

Le gène $B R C A 1$ semble impliqué dans les formes familiales de susceptibilité aux cancers du sein et de l'ovaire mais peu dans les formes sporadiques. Sur 32 cas de tumeurs mammaires et 12 cas de tumeurs ovariennes sporadiques, avec un phénomène de perte d'hétérozygotie dans la région du bras long du chromosome 17 portant le gène $B C R A 1$, des mutations de ce gène n'étaient observées que 4 fois, et étaient également retrouvées dans les tissus normaux, indiquant par là qu'il s'agissait d'une anomalie germinale. Ces résultats suggèrent, soit que d'autres gènes de susceptibilité, modifiés dans les formes sporadiques, existent à proximité du gène $B C R A 1$, soit qu'une perte somatique d'un des allèles $B C R A 1$, éventuellement en collaboration avec une autre anomalie, peut être impliquée dans ces formes sporadiques de cancer [3].

La découverte du gène $B R C A l$ est symbolique à plus d'un titre. Elle est un exemple de ce que la compétition entre des équipes académiques, même prestigieuses, et des firmes privées est biaisée dès lors que son objet justifie que soient consentis des investissements financiers considérables. Naturellement, et comme cela se pratique dans tous les pays du monde lorsqu'un gène dont la fonction est connue et qui pourrait avoir une utilisation économiquement intéressante est isolé, des demandes de brevets ont été déposées sur le gène $B R C A 1$ : brevet de composition de substance et brevet de procédé, couvrant le gène lui-même, la protéine qui lui correspond, leur utilisation pour la mise au point de tests diagnostiques et pour des développements de procédés thérapeutiques. C'est que, compte tenu de la fréquence de la maladie, les perspectives des marchés de tels procédés 
ou de tels produits sont considérables, pouvant se chiffrer à plusieurs milliards de dollars dans le monde rien que pour le test diagnostique.

C'est dire que la course est maintenant lancée pour un deuxième gène de susceptibilité appelé $B C R A 2$ localisé sur le bras long du chromosome 13 (intervalle 13q12-13) qui vient d'être localisé par une équipe anglaise [4]. Contrairement à $B C R A 1, B C R A 2$ semblerait prédisposer des familles au cancer du sein mais non pas au cancer de l'ovaire. A eux deux, les gènes $B R C A 1$ et BRCA2 pourraient rendre compte de plus des deux tiers des formes familiales du cancer du sein.

Plusieurs lectures sont possibles de ces récents développements spectaculaires dans le domaine de la génétique des cancers. On peut se réjouir de ce que l'importance des enjeux économiques ait justifié des investissements considérables accélérant la recherche et la découverte. Dans le même temps, il faut observer que la situation des équipes académiques, souvent à l'origine des observations permettant ces découvertes, devient extrêmement inconf ortable puisqu'il leur est tout à fait impossible d'investir des moyens comparables, à un ordre de grandeur près, à ceux mis en œuvre par ces sociétés privées drainant des fonds sur le marché des capitaux. Ainsi peut-on observer qu'une partie de l'énergie d'importantes équipes de chercheurs est stérilisée dans des compétitions un peu sans espoir.

Une autre lecture est de se poser la question des conséquences de ces découvertes génétiques en terme de santé publique et d'évolution des dépenses de santé. Pour l'instant, la découverte des gènes de susceptibilité aux différentes formes du cancer du côlon ([5] et $\mathrm{m} / \mathrm{s} n^{\circ} 11$, vol. 10 , p. 1178) et au cancer du sein offrent des perspectives de prédiction et non point de traitement, et cette situation a des chances de durer un certain temps. Dans le cas des gènes de susceptibilité au cancer du côlon, qui sont tous impliqués dans la réparation des mésappariements de l'ADN, la découverte de la nature du gène n'oriente guère vers des stratégies thérapeutiques mais indique plutôt pourquoi celles-ci seront bien difficiles à mettre en œuvre. Cependant, les tests diagnostiques seront bénéfiques s'ils permettent, dans des fa- milles à risques, de rassurer ceux qui n'ont pas hérité de l'allèle morbide et, en général, s'ils débouchent sur une surveillance permettant de traiter plus tôt des cancers et donc d'augmenter les chances de succès. Encore faut-il qu'il soit bien clair qu'un diagnostic précoce est un gage particulier de succès du traitement, ce qui semble aujourd'hui au moins douteux dans le cas des cancers du sein. De ce fait, la chirurgie prophylactique (mammectomie et ovariectomie dans le cas des gènes BRCA, colectomie totale dans le cas des gènes de susceptibilité au cancer du côlon) peut légitimement être proposée aux sujets porteurs du gène dans les familles à risque. Par ailleurs, l'intérêt commercial des firmes qui se seront vu attribuer les brevets sur l'utilisation de ces tests diagnostiques sera évidement d'en promouvoir la diffusion la plus large, ce qui ne devrait pas être trop difficile auprès d'une population déboussolée et, d'une manière beaucoup plus consciente, auprès des systèmes d'assurance maladie et vie. $\mathrm{Si}$ cette promotion est un succès, sans même discuter la redoutable question de la gestion de la connaissance d'une susceptibilité à des cancers que l'on ne sait pas éviter, elle aboutira vraisemblablement à une explosion des dépenses de santé pour des bénéfices faibles en terme de vies sauvées. L'aventure de la recherche génétique est étourdissante et exaltante. Les généticiens et les médecins en général sauront-ils, tout en participant à cette exaltation du progrès scientifique, en éviter l'étourdissement afin d'envisager sereinement les conséquences possibles de l'utilisation des outils ainsi créés et, si possible, les conditions de leur maîtrise?

A.K.

1. Hall JM, Lee MK, Newman B, Morrow JE, Anderson LA, Huey B, King MC. Linkage of early-onset familial breast cancer to chromosome 17q21. Sciences ; 256, 1684-9.

2. Miki Y, Swensen J, Shattuck-Eidens D... (44 auteurs au total)... Skolnick MH. A strong candidate for the breast and ovarian cancer susceptibility gene BRcAl. Science 1994; $226: 66-71$.

3. Futreal PA, Liu Q, Shattuck-Eidens D... (26 auteurs au total)... Wiseman R. BCRA 1 mutations in primary breast and ovarian carcinomas. Science $1994 ; 226$ : $120-2$.

4. Wooster, et al. Science 1994 (sous presse).

5. Radman M, Taddei F, Halliday J. Correction des erreurs dans l'ADN : de la génétique bactérienne au mé canisme de prédisposition héréditaire aux cancers chez l'homme. médecine/sciences $1994 ; 10$ : 1024-30.

\section{BRÈVES}

Les protéasomes à composition variable. Les protéasomes sont des unités multicatalytiques qui dégradent les protéines endogènes par des mécanismes dépendants de l'ubiquitine et de l'ATP. Ils ont été très conservés au cours de l'évolution et les mammifères supérieurs ont même adapté ce système ubiquitine/protéasomes à la lutte contre les agents pathogènes in tracellulaires. Sous l'action de l'interféron $\gamma$ (IFN $\gamma)$, l'expression de deux sous-unités non obligatoires du protéasome, LMP2 et LMP7, est très augmentée et le spectre d'activité des protéasomes change pour fournir des peptides aux caractéristiques nouvelles $\left(\mathrm{m} / \mathrm{s} n^{\circ} 11\right.$, vol. 9, p. 1284). En plus d'un changement de spécificité pour les substrats peptidiques, la composition même du protéasome peut varier. En effet, l'IFN $\gamma$ provoque une diminution de l'expression de deux autres sous-unités présentant des similitudes importantes avec LMP2 et LMP7. L'IFN $\gamma$ induirait le remplacement de ces deux sous-unités par LMP2 et LMP7, favorisant ainsi la production de peptides plus efficacement présentés aux lymphocytes T cytotoxiques [1, 2]. L'importance de LMP7 vient aussi d'être démontrée in vivo à l'aide de souris dont le gène LMP7 est invalidé [3]. Le niveau d'expression du complexe majeur d'histocompatibilité de classe I est faible chez ces souris ainsi que la capacité de présentation d'antigènes endogènes. Qu'elle agisse directement sur la genèse des peptides, ou indirectement en rapprochant physiquement les protéasomes des complexes TAP, transporteurs de peptides vers le réticulum endoplasmique, il est clair que cette protéine LMP7 joue un rôle primordial dans l'approvisionnement en peptides.

[1. Akiyama K, et al. Science 1994 ; 265: 1231-3.]

[2. Früh K, et al. EMBO J $1994 ; 13$ : 3236-44.]

[3. Fehling M, et al. Science 1994 ; 265 : 1234-7.] 